\title{
Direct sensing of coke deposits on fixed bed catalysts in refinery processes
}

\author{
N. Müller ${ }^{1,2}$, A. Jess ${ }^{1}$, R. Moos ${ }^{2}$, \\ ${ }^{1}$ Chemical Engineering, ${ }^{2}$ Functional Materials, \\ University of Bayreuth, 95440 Bayreuth, Germany
}

\begin{abstract}
The activity of heterogeneous catalysts in refinery processes is substantially influenced by formation of coke deposits. This study expands an in-situ method for direct determination of the coke loading in fixed bed catalysts by utilizing suspension balance techniques. Here, it is investigated whether it is possible to measure the coke content during coking and regeneration. Applied to a technical relevant $\mathrm{Al}_{2} \mathrm{O}_{3}$-based $\mathrm{Cr}_{2} \mathrm{O}_{3}$-catalyst, it is shown that the electrical impedance of a catalyst pellet is strongly correlated with its degree of coke loading.
\end{abstract}

Keywords: coke sensor, catalytic reforming

\section{Introduction and Experimental}

In several refinery and petrochemical processes, the catalyst deactivates by coke formation, e.g. during reforming of gasoline or dehydrogenation of paraffins [1]. In contrast to soot in exhaust gas streams, where soot is already present in the gas flow, in refinery catalysts, the coke usually forms inside the highly porous catalyst particle [2, 3].

Therefore, a sensor was investigated that is suitable to directly monitor the degree of coke loading of catalyst pellets in refineries. The key issue to be addressed is whether the electrical properties of a single catalyst pellet correlates with its coke loading. The investigations were conducted with pellets of typical reforming and dehydrogenation catalysts. The catalyst consists of porous $\mathrm{Al}_{2} \mathrm{O}_{3}$ pellets as support material that was coated with $\mathrm{Cr}_{2} \mathrm{O}_{3}$ as the active component. Furthermore, Pt- or Pdcoated systems were successfully tested. One representative pellet in the fixed bed catalyst serves as sensing element. Its impedance is measured with a setup as shown in Fig 1.

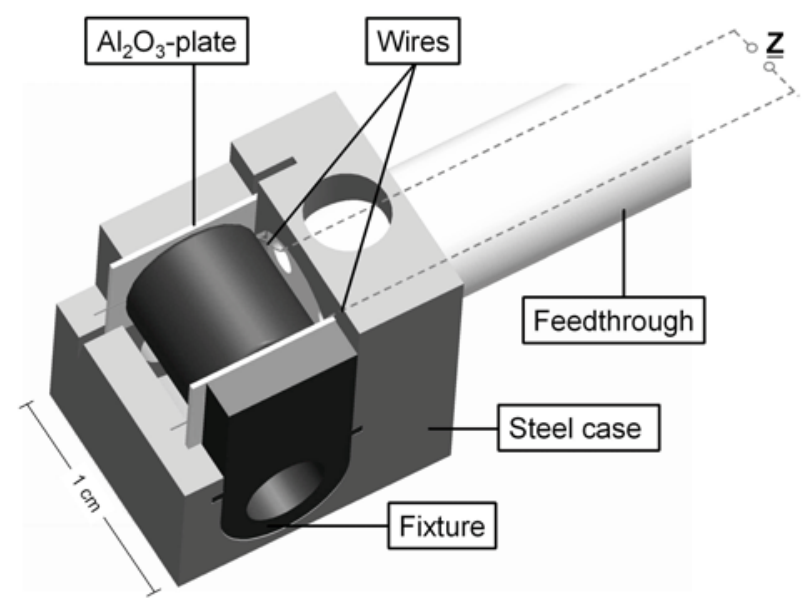

Fig. 1: $C A D$ picture of the coke sensor 
In Fig 2, the schematic experimental setup, which contains all components of a real refinery system, is shown. An inert gas flow was saturated with propylene to get a fast coke formation. In a tubular reactor coupled with a suspension balance, the gas flow passes the catalyst pellets and deactivates them by coke formation. The impedance spectrum of the sensor was measured continuously between $100 \mathrm{~Hz}$ and $1 \mathrm{MHz}$. The coke loading was measured in a suspension balance. Temperatures, pressures and gas concentrations and space velocities were similar to real applications.

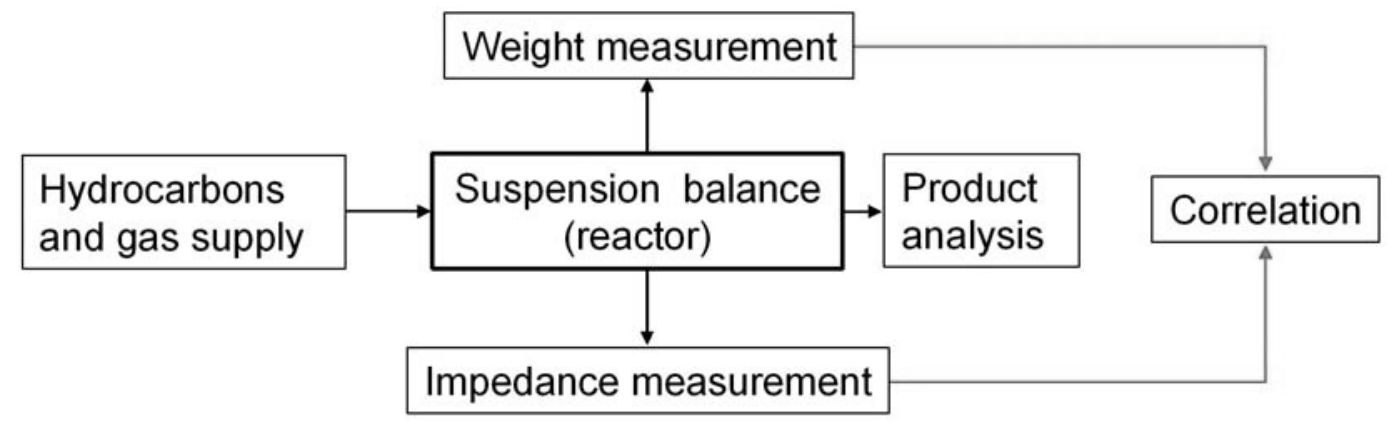

Fig. 2: Schematic experimental setup

\section{Results and Discussion}

In initial tests, it was found out that data taken at $10 \mathrm{kHz}$ are the best compromise between a good signal amplitude and a correlation with the coke loading. Fig. 3 shows the simultaneous measurement of the

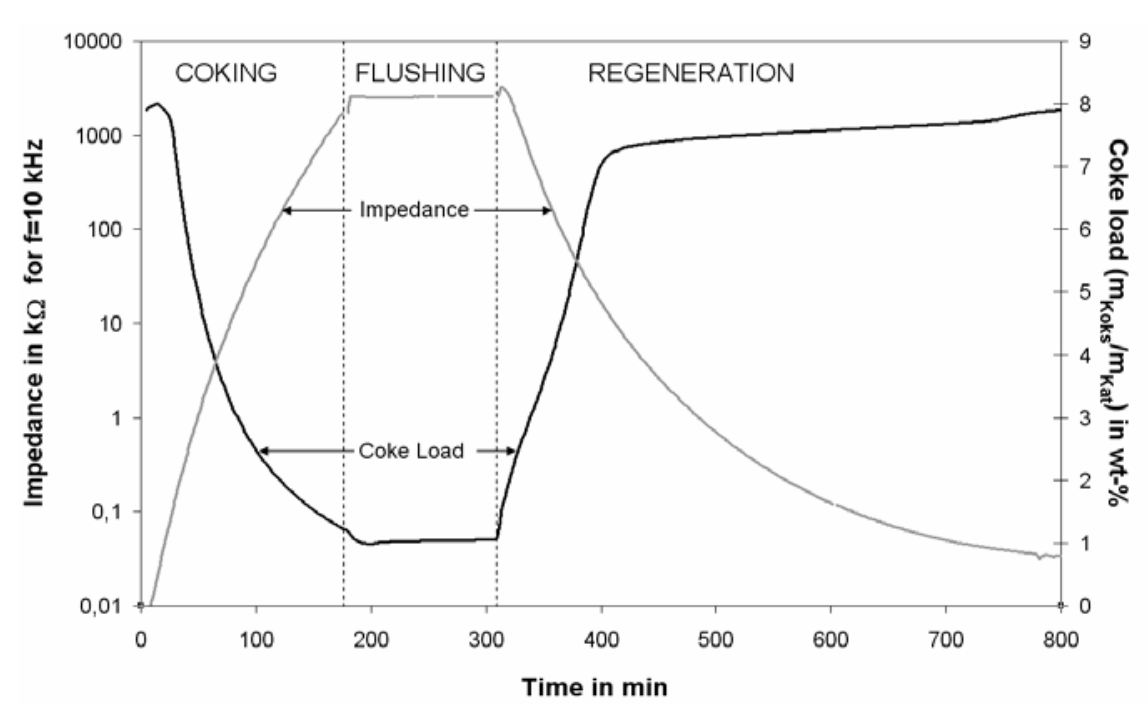

Fig. 3: Coke load and impedance vs time change in impedance and content of coke depending on the experimental time. While increasing the amount of coke the impedance decreases. After three hours the coking where stopped and the reactor was flushed to avoid reactions between the model hydrocarbon and the oxygen. Then, the regeneration starts and the behavior of the sensor changes. To visualize the interrelationship in Fig 4, the coke loading is plotted vs. the absolute value of impedance at $f=10 \mathrm{kHz}$ during coking and regeneration. For less than one weight percent coke load, the coking signal is dominated by the gas sensitive character of the semiconducting $\mathrm{Cr}_{2} \mathrm{O}_{3}$. The increase of the coke mass is observable only by the phase signal in Fig 4 which changes from almost $70^{\circ}$ (capacitive character dominates the signal) to $0^{\circ}$ (ohmic behavior). Above two weight percent coke load, a monotonous behavior is observed, i.e. an increased coke loading leads to a reduced electrical impedance. It is assumed that the coke loading goes along with the formation of conductive paths through the catalyst, which are responsible for the decreasing impedance. The regeneration signal shows a 
hysteresis curve. This is caused by the different reaction processes of coke formation and coke oxidation. The coke formation is limited by the chemical kinetics, whereas the oxidation is limited by pore diffusion. So the coke is formed homogeneously distributed in the catalyst pellet, whereas the regeneration goes from outside the particles to the center. The phase signal shows these differences clearly. The uniform coking causes a wide ohmic dominated range, whereas the conductive paths where cut off by the hard conditions during regeneration. If the regeneration temperatures were reduced the both curves become similar.

\section{Conclusion}

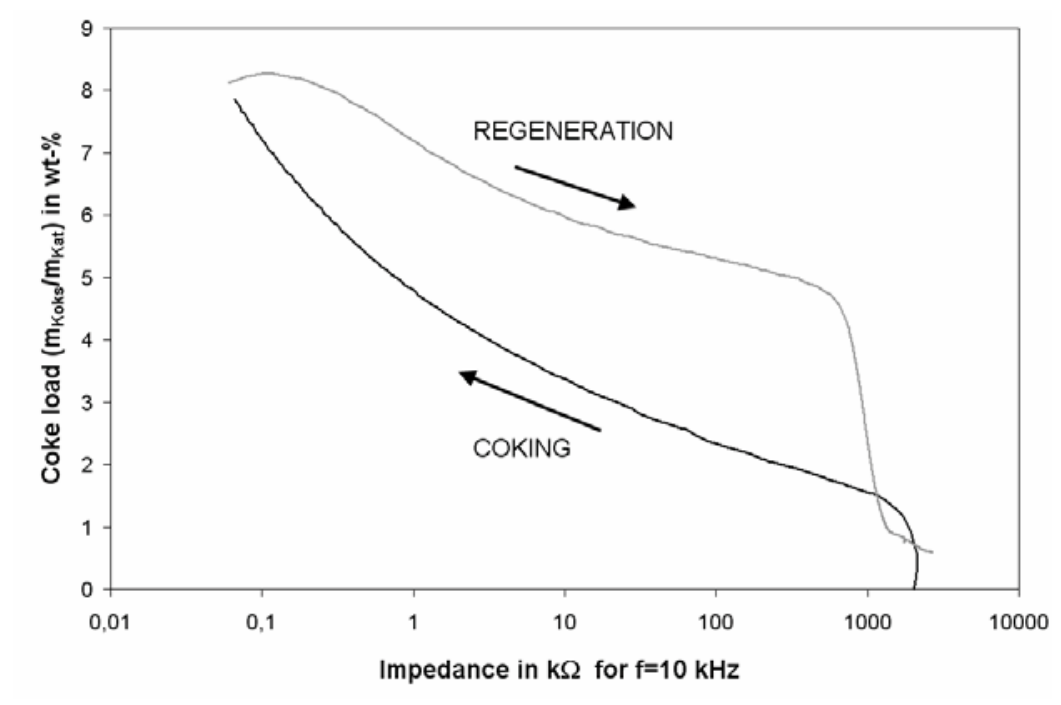

It was possible to determine insitu the coke content of a heterogeneous catalyst under reaction conditions by impedance measuring of single catalyst pellets. Furthermore, the coke content was measured during coking and regeneration and a correlation between impedance data and coke content was found. Future investigations will focus on the sensor housing and on investigating long-term effects with the goal of real refinery applications. In addition, correlations between theoretical models and the impedance results are under study.

\section{References}

1. C. Kern, Regeneration of coked fuel-reforming catalyst, Dissertation, Univ. Bayreuth, 2003

2. J. Riegel, $5^{\text {th }}$ Int. Exhaust Gas and Particulate Emissions Forum, Ludwigsburg, 19.-20.2.2008, 85-96

3. C. H. Bartholomew, Mechanism of catalyst deactivation, Applied Catalysis A: General 212, 2001, 17-60 